\title{
STRUCTURE OF SCABIOSIDE F
}

\author{
V. G. Bukharov and V. V. Karlin
}

Khimiya Prirodnykh Soedinenii, Vol. 6, No. 3, pp. 372-373, 1970

UDC $547.597+547.918$

Scabioside $\mathrm{F}$, a triterpene glycoside with $\mathrm{mp} 232-235^{\circ} \mathrm{C},[\alpha]_{\mathrm{D}}^{20}-21^{\circ}$ (c 5.2 , pyridine), was isolated from Patrinia scabiosofolia Fisch, et Link [1]. On acid hydrolysis it decomposes into oleanolic acid, D-glucose, L-arabinose, D-xylose, and L-rhamnose. According to its molecular weight (1160) scabioside $F$ is a pentaoside. On alkaline hydrolysis on an anion-exchange resin [2], scabioside $F$ is converted into scabioside $B$, i.e. , into oleanolic acid $3-\mathrm{O}-\beta-\mathrm{D}$-glucopyranosyl- $(1 \rightarrow 4)-\alpha$-L-arabinopyranoside

Exhaustive methylation of scabioside $\mathrm{F}$ and subsequent cleavage of the resulting permethylate with $\mathrm{HCl}$ gave 2, 3, 4, 6-tetra-O-methyl-D-glucopyranose, 2,3-di-O-methyl-L-arabinopyranose, 2, 3-di-O-methyl-D-xylopyranose, and a dimethyl-L-rhamnose. Of the three possible dimethyl-L-rhamnose derivatives $(2,3-, 2,4-$, or 3,4-di-O-methyl) we chose 2,4-di-O-methyl-L-rhamnopyranose. The correctness of this choice is confirmed by the results of the periodate oxidation of scabioside $F$, in which all the monosaccharide residues with the exception of the L-rhamnose are destroyed.

Reductive cleavage of the permethylate of scabioside $\mathrm{F}$ using lithium aluminum hydride gave a methylated trisaccharide which decomposed on acid hydrolysis into 2, 3,4-tri-O-methyl-D-xylopyranose, 2, 4-di-O-methyl-Lrhamnopyranose, and 2,3-di-O-methyl-D-xylitol.

Consequently, one xylose is attached directly to the carboxyl of oleanolic acid and the other is present in a trisaccharide chain in which, as is shown by calculations based on the difference in the molecular rotations between scabiosides $\mathrm{E}[1]$ and $\mathrm{F}$, the terminal xylose has a $\beta$-bond. The complete coincidence of the configurations of the analogous glycoside centers of the two scabiosides is also confirmed by the results of calculations by Klyne's method. Thus scabioside $\mathrm{E}$ is a genetic precursor of scabioside $\mathrm{F}$. On the basis of the above, scabioside $\mathrm{F}$ must be ascribed the following structural formula

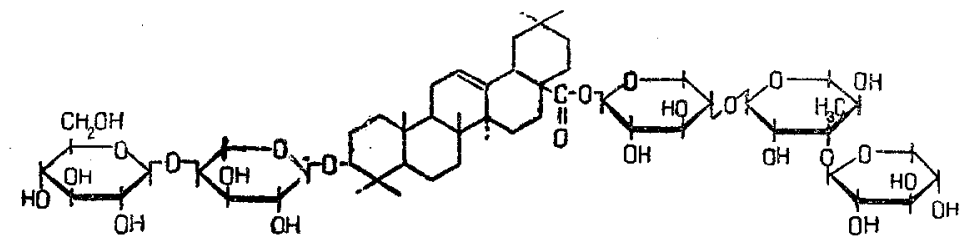

REFERENCES

1. V. G. Bukharov and V. V. Karlin, KhPS [Chemistry of Natural Compounds], 6, 211, 1970.

2. V. G. Bukharov, V. V. Karlin, and V. A. Talan, KhPS [Chemistry of Natural Compounds], 3, $17,1967$.

3. V. G. Bukharov, V. V. Karlin, and T. N. Sidorovich, KhPS [Chemistry of Natural Compounds], 6, 69, 1970.

21 October 1969

Arbuzov Institute of Organic and Physical Chemistry, AS USSR 